IGC-08/11-5

\title{
Consistent Loop Quantum Cosmology
}

\author{
Martin Bojowald* \\ Institute for Gravitation and the Cosmos, \\ The Pennsylvania State University, \\ 104 Davey Lab, University Park, PA 16802, USA
}

\begin{abstract}
A consistent combination of quantum geometry effects rules out a large class of models of loop quantum cosmology and their critical densities as they have been used in the recent literature. In particular, the critical density at which an isotropic universe filled with a free, massless scalar field would bounce must be well below the Planck density. In the presence of anisotropy, no model of the Schwarzschild black hole interior analyzed so far is consistent.
\end{abstract}

Aside from detailed technical constructions, one of the main achievements of loop quantum gravity [1, 2, 3] is to provide a framework for discrete dynamical geometries. Loop quantum cosmology [4 takes these ingredients and applies them to expanding universes or black hole models. In this way, effects of quantum physics as well as quantum geometry can be explored in detail. This is a specific realization of a general issue which has been discussed recurrently in the context of discrete models of gravity [5, 6, 7, 8, 9]. Loop quantum gravity makes many of the older considerations more specific, but since neither its complete form nor the precise transition to loop quantum cosmology has been hammered out as of now, the dynamics of loop quantum cosmology cannot be unique. Current ignorance must be parameterized so that at least qualitative implications can be found. In particular, all corrections from quantum physics and quantum geometry must be analyzed before reliable and robust conclusions can be drawn. Here we consider quantum geometry in regimes where its implications are dominant over genuine quantum corrections, and provide a consistent combination of its two main effects: holonomy corrections but also inverse volume corrections which have often been ignored. As we will see, this combination leads to tight consistency conditions which rule out parameter choices made so far in cases where only holonomy corrections were considered. Quantum back-reaction effects, which still remain to be fully derived following the methods of [10, 11], will not be required for our analysis.

We start with a discussion of isotropic models, which classically have only one invariant scale: the Hubble distance $\mathcal{H}^{-1}=a / \dot{a}$ (a dot meaning a derivative by proper time). If we

*e-mail address: bojowald@gravity.psu.edu 
consider a fixed region $\mathcal{V}$ in our isotropic space, a second scale $a^{3} V_{0}$ arises which is independent of coordinates but depends on the coordinate size $V_{0}$ of the region chosen. These two scales provide the classical canonical pair, $\left\{\mathcal{H}, V_{0} a^{3}\right\}=4 \pi G$ with the gravitational constant $G$.

Quantum geometry effects are expected to arise if the Hubble scale is Planckian, $\mathcal{H}^{-1} \sim \ell_{\mathrm{P}}$. Technically, this is realized in loop quantum gravity because holonomies, which are nonlinear functions of $\dot{a}$ and $a$, replace the classical Hubble parameter in the gravitational Hamiltonian [12]. But also the intrinsic spatial geometry, which depends on a but not on $\dot{a}$, implies corrections when all of space is made of discrete patches whose size is near Planckian. (By "patch" we will mean the smallest building block of a discrete geometry.) This arises technically because inverse patch sizes appear in Hamiltonians, and their quantizations differ from classical values for small enough patches [13, 14]. However, in contrast to the invariant scale $\dot{a} / a$, there is no invariant measure for the spatial geometry in a purely isotropic setting: the scale factor $a$ is coordinate dependent while the coordinate independent size $V_{0} a^{3}$ depends on the region $\mathcal{V}$ chosen. This is the reason why inverse volume corrections have not been fully realized in minisuperspace models so far, and most often were ignored.

The patch size refers to an underlying discrete state giving rise to the expanding universe. To include this effect in a pure minisuperspace model, extra input is thus required from the full theory, which must guide the construction of homogeneous models. Fortunately, this is possible in a heuristic mean-field picture [15] where a mean-field describing the underlying discrete structure is used. As always with mean-field approximations, the precise way in which the field arises from a fundamental theory may be very difficult to derive. In lieu of a detailed derivation, however, there are often consistency conditions which can help to reduce the a-priori freedom involved in the choice of mean-field behaviors. This turns out to be the case for loop quantum cosmology, too.

We then have a patchwork structure describing the isotropic classical geometry in an atomic manner. Instead of the classical size $a^{3} V_{0}$, we can write $\mathcal{N} v$ where $v$ is the mean size of a patch and $\mathcal{N}$ is their number in the region $\mathcal{V}$. Since both products are supposed to give the size of the same region, they must equal each other:

$$
\mathcal{N}(t) v(t)=a(t)^{3} V_{0}
$$

In this equation, we have already accounted for the main result from loop quantum gravity employed here: the fact that any dynamics proposed for the full theory [16, 13, 17] must change the values of discrete contributions to volume; $v(t)$ must depend on time as nodes representing the atomic volume elements are being excited. In general, also the number of patches $\mathcal{N}(t)$ is a function of time, and the product of both functions provides the time dependence of the scale factor $a(t)$. (For the current purposes it is irrelevant what time variable is used. In explicit constructions, this could be an internal time such as a matter field.)

As an important consequence, we see that the expansion of an isotropic universe in loop quantum cosmology is described by two time-dependent functions, $\mathcal{N}(t)$ and $v(t)$, 
rather than one classical function $a(t)$. Thus, there is more freedom in the underlying dynamics which is related to the refinement behavior of an underlying discrete state. By a pure minisuperspace quantization, which starts from the classical $a$ and turns it into an operator, this freedom cannot be constrained but rather emerges in the form of quantization ambiguities in the reduced Hamiltonian. The detailed form of the dynamical change of $\mathcal{N}$ and $v$ can in principle be derived from the full theory, or be restricted by analyzing the implied phenomenology of models. (It is not uncommon that additional parameters and functions not seen classically arise from a quantum or microscopic treatment. In cosmological models, for instance, a similar behavior has been observed in [18].)

Quantum geometry is discrete but local, and thus corrections to the classical geometry and its dynamics depend only on the microscopic patch size $v$ rather than the macroscopic number $\mathcal{N}$ of patches. (From a classical perspective, this behavior may not seem local if a discrete patch such as the geometry given by a single spin network vertex is viewed as a representation of the continuum distribution in a neighborhood. But quantum geometry is local in the sense that geometrical operators only refer to sums over single patch contributions, a behavior which we will call "patch-local" in what follows.) This already implies that all consistent implementations of quantum corrections must be independent of the size $V_{0}$ of the region, as this is a parameter which determines $\mathcal{N}$ but does not affect $v$.

We first address holonomy corrections which are easier to construct, although also here extra input is needed compared to the original and direct quantization used in loop quantum cosmology [19]. Holonomies are non-linear functions of a connection which in isotropic models is simply proportional to $\dot{a}: \tilde{c}=\gamma \dot{a}$ with the Barbero-Immirzi parameter $\gamma$ [20, 21]. In a patch-local holonomy, the connection appears in a line integral along a curve of the (linear) patch size $\ell_{0}$. In an isotropic setting, holonomies are thus functions of $\ell_{0} \tilde{c}=\gamma L \dot{a} / a$ where $L=\ell_{0} a$ is the geometrical and coordinate independent length of the curve (or its co-moving size).

The number of patches of coordinate size $\ell_{0}^{3}$ in a region of size $V_{0}$ is given by $\mathcal{N}=V_{0} / \ell_{0}^{3}$. Together with (1), this implies that $L=\ell_{0} a=\left(V_{0} / \mathcal{N}\right)^{1 / 3} a=v^{1 / 3}$ is directly related to the microscopic patch size $v$. Thus, holonomy corrections, which arise from deviations of non-linear functions such as $\sin (\gamma L \dot{a} / a)$ from the linear $\gamma L \dot{a} / a$, are independent of the size $V_{0}$, as required. Below we will see that this is also true for inverse volume corrections.

Before discussing inverse triad corrections and their mutual consistency with holonomy corrections, we emphasize the dynamical role of $L(t)=v(t)^{1 / 3}$. In the original formulation of loop quantum cosmology [19, 22], it was assumed that $\ell_{0}$ is constant as the universe expands. This is the most straightforward assumption in a pure minisuperspace construction, but it was clear that this cannot describe long phases of expansion: a constant $\ell_{0}=\left(V_{0} / \mathcal{N}\right)^{1 / 3}$ implies that the number of patches in the co-moving region $\mathcal{V}$ is constant. As the geometrical size of this region is expanded by the scale factor, a constant number of patches means that patch sizes are expanded to macroscopic values. This would clearly be in conflict with a large universe free of noticeable quantum geometry effects. (The correspondence between constant $\ell_{0}$ and a constant number of patches is also demonstrated by the two-patch model of [23].) 
To address this problem, an improvised version of the dynamics was proposed in [24], based on the ad-hoc assumption that low-lying eigenvalues of the full area spectrum should determine quantization parameters of minisuperspace models. This assumption is ad-hoc because the area operator plays no role in full constructions of Hamiltonian constraints or their solutions. One could think that it somehow arises in a gauge-fixing procedure which leads one from the full theory to an isotropic model, but no such construction is known. (The use of the area operator, which is not a physical observable, was criticized in [25]. However, the area is used when constructing the constraint and before solving it; non-observables can certainly appear in this step just as $\dot{a} / a$, which is not a constant of motion, appears in the classical constraint.) Despite of these shortcomings, the procedure implied that it is not $\ell_{0}$ which is constant but $L=\ell_{0} a=v^{1 / 3}$. This means that the microscopic patch size is constant in this version, and correspondingly the number of patches increases in an expanding universe. In this way, strong quantum geometry effects in a large universe can be avoided for suitable parameter choices. However, the values proposed in [24], motivated by the smallest non-zero area eigenvalues, turn out to be in conflict with inverse volume corrections, to which we turn now.

Inverse volume corrections arise because the patch volume $v$, when quantized, becomes an operator with discrete spectrum containing zero [26, 27]. Such an operator does not have a densely defined inverse, but an inverse patch volume is needed in most Hamiltonians. One can construct operators with the correct classical limit given by the inverse based on identities such as

$$
i e^{i \ell_{0} c / V_{0}^{1 / 3}}\left\{e^{-i \ell_{0} c / V_{0}^{1 / 3}},|p|^{3 r / 2}\right\}=4 \pi \gamma G \frac{\ell_{0}}{V_{0}^{1 / 3}}|p|^{3 r / 2-1} \operatorname{sgn}(p)
$$

for the canonical pair $c=V_{0}^{1 / 3} \tilde{c}=V_{0}^{1 / 3} \gamma \dot{a}$ and $|p|=V_{0}^{2 / 3} a^{2},\{c, p\}=8 \pi \gamma G / 3$, underlying isotropic loop quantum cosmology. For $0<r<2 / 3$, the right hand side gives an inverse power of the scale factor, while no inverse is needed on the left. This gives rise to well-defined quantizations, which are part of a general procedure [14], and whose specific constructions in isotropic models all follow the derivations in [28] to which we refer for further details.

Since the quantized holonomy $\exp \left(i \ell_{0} c / V_{0}^{1 / 3}\right)$ becomes a shift operator in $p$ and the Poisson bracket in (2) becomes a commutator, the quantization of inverse volume provides a discrete approximation of the derivative involved in the classical Poisson bracket. The derivative $\mathrm{d}|p|^{3 r / 2} / \mathrm{d} p$ on the right hand side of (2) is then replaced by a difference $\left(|p+\Delta p|^{3 r / 2}-|p-\Delta p|^{3 r / 2}\right) / 2 \Delta p$ where $\Delta p$ depends on the precise implementation of the quantization scheme and is typically related to the Planck length. (Numerical studies of the volume spectrum in the full theory, whose results will shed light on which precise values are to be expected, are on-going [29, 30, 31, 32.) Deviations of the difference from the derivative give rise to inverse volume corrections.

The form of $\Delta p$ is important to demonstrate the consistency of inverse volume corrections, i.e. independence of $V_{0}$, and to estimate their size. Inverse volume corrections as they have been used in most isotropic models so far correspond to using the total volume 
$V=a^{3} V_{0}$ of the region $\mathcal{V}$ and approximating its derivative by differences of the form $V \pm \ell_{\mathrm{P}}^{3}$. This has two consequences: (i) inverse volume corrections of this form are extremely tiny when the total volume is much larger than the Planck volume, and (ii) such corrections depend on the region $\mathcal{V}$ and its coordinate size $V_{0}$ because $V$ does. The first property led [24] to conclude that inverse volume corrections can safely be ignored compared to holonomy corrections, but the $V_{0}$-dependence remained unexplained; in fact, it points to an inconsistency in the derivations used which cannot simply be eliminated by ignoring inverse volume corrections.

In the present context one can see a third problem with such a treatment of inverse volume corrections: if quantizations of the inverse volume depend on the total volume of a region, resulting operators cannot be patch-local. Inverse volume operators appearing e.g. in energy densities would depend on the full volume of a region $\mathcal{V}$ which can be macroscopic. Although this may not appear as a problem in an isotropic context where homogeneity identifies local and global properties, it is clear that such quantizations cannot arise in a model consistently related to what we know from the full theory. As already mentioned, full Hamiltonians have contributions which are patch-local and depend on the patch-volume $v$ but not on the number of patches $\mathcal{N}$ or the total volume $V$. This has two important consequences: (i) consistent implementations of inverse volume corrections do not depend on $V_{0}$ since $v$ does not, and (ii) they are much larger than naively expected because $v$ is much smaller than $V$ and differences $v \pm \ell_{\mathrm{P}}^{3}$ deviate much more from infinitesimal displacements of $v$ than $V \pm \ell_{\mathrm{P}}^{3}$ does from displacements $\mathrm{d} V$ of the large $V$. That inverse volume corrections are artificially suppressed in direct minisuperspace quantizations has also been discussed in the appendix of [33].

Consistent inverse volume corrections imply correction functions $\alpha\left(L / \ell_{\mathrm{P}}\right)$ at all places where inverse densitized triad components are used in a classical Hamiltonian, such as in the kinetic term of a matter Hamiltonian [14]. The precise functional form of $\alpha$ is subject to quantization ambiguities [34, 35, 36], but is restricted by anomaly cancellation conditions [37]. In all cases, $\alpha$ deviates strongly from the classical value $\alpha=1$ when its argument is of the order one or smaller; for large values of $L / \ell_{\mathrm{P}}$ the classical limit is approached asymptotically. Importantly, correction functions $\alpha\left(L / \ell_{\mathrm{P}}\right)$ are to be evaluated at $L / \ell_{\mathrm{P}}$ rather than the much larger and $V_{0^{-}}$dependent $V^{1 / 3} / \ell_{\mathrm{P}}$. There is thus no $V_{0^{-}}$ dependence, and quantum geometry effects from inverse volume operators are much more pronounced than expected. In this way, valuable consistency conditions can arise. In particular, for holonomy corrections to be small in classical regimes we require $\ell_{0} \tilde{c}=$ $\gamma L \dot{a} / a \ll 1$, while small inverse volume corrections require $v^{1 / 3} / \ell_{\mathrm{P}}=L / \ell_{\mathrm{P}} \gg 1$. For a given classical geometry, we thus have an upper as well as a lower bound on $L$, which leaves only a finite range of allowed choices.

To make this quantitative, we derive the main effect of holonomy corrections in the current setting, and compare parameter values with what is consistent with inverse volume corrections. The Hamiltonian constraint is the main place where corrections enter, and 
holonomy corrections imply a form

$$
C=-\frac{3}{8 \pi G \gamma^{2}} \frac{V_{0}^{2 / 3}}{\ell_{0}^{2}} \sqrt{|p|} \sin ^{2}\left(\ell_{0} c / V_{0}^{1 / 3}\right)+|p|^{3 / 2} \rho=0
$$

with a matter density $\rho$. These terms strictly refer to operators as they appear in a quantization of the Hamiltonian constraint, but they can also be considered as the tree-level approximation to the quantum constraint operator. Our considerations will not require deep quantum regimes as they are, e.g., encountered around classical singularities, where such tree-level equations would be unreliable due to the presence of additional quantum corrections [38, 39]. The tree-level constraint then implies equations of motion with treelevel corrections from quantum geometry. We will only require the equation

$$
\dot{p}=\{p, C\}=2 \sqrt{p} \frac{V_{0}^{1 / 3}}{\gamma \ell_{0}} \sin \left(\ell_{0} c / V_{0}^{1 / 3}\right) \cos \left(\ell_{0} c / V_{0}^{1 / 3}\right)
$$

which can be written as

$$
\begin{aligned}
\left(\frac{\dot{a}}{a}\right)^{2} & =\left(\frac{\dot{p}}{2 p}\right)^{2}=\frac{1}{\gamma^{2} \ell_{0}^{2} a^{2}} \sin ^{2}\left(\ell_{0} c / V_{0}^{1 / 3}\right)\left(1-\sin ^{2}\left(\ell_{0} c / V_{0}^{1 / 3}\right)\right) \\
& =\frac{8 \pi G}{3} \rho\left(1-\frac{8 \pi G}{3} \gamma^{2} \ell_{0}^{2} a^{2} \rho\right)
\end{aligned}
$$

where we used the Hamiltonian constraint (3) to express $\sin ^{2}\left(\ell_{0} c / V_{0}^{1 / 3}\right)$ in terms of $\rho$. Defining the critical density

$$
\rho_{\text {crit }}=\frac{3}{8 \pi G \gamma^{2} L^{2}}
$$

we thus have the tree-level Friedmann equation [40]

$$
\left(\frac{\dot{a}}{a}\right)^{2}=\frac{8 \pi G}{3} \rho\left(1-\frac{\rho}{\rho_{\text {crit }}}\right)
$$

whose behavior is determined by the patch size $L$ (see also [41] for the derivation with general $L$ ). The meaning of the critical density $\rho_{\text {crit }}$ can be seen in a model where the only matter ingredient is a free, massless scalar: In this case the tree-level equation is an exact effective equation [42] and can be used even in deep quantum regimes. When $\rho=\rho_{\text {crit }}$, the scale factor reaches a minimum and the universe bounces. For other matter there are additional quantum corrections [39], and even the tree-level form can change if higher order holonomy corrections are included which are possible due to quantization ambiguities [43]. The bounce has reliably been demonstrated only for a free massless scalar, but the critical density can nonetheless be used more generally as a measure for the size of holonomy corrections.

It is hard to derive $L$ from an inhomogeneous state in full loop quantum gravity, but one can easily check the mutual consistency of any value for $\rho_{\text {crit }}$ in holonomy corrections 
with the corresponding implications of $L$ in inverse volume corrections. In the case of [24], which is the main specific model analyzed recently, we have a constant $L$ which, by reference to the full area spectrum, gives rise to a critical density near the Planck density: $\quad \rho_{\text {crit }} \sim \rho_{\mathrm{P}}=1 / G \ell_{\mathrm{P}}^{2}$. With (6) we obtain $\gamma L \sim \sqrt{3 / 8 \pi} \ell_{\mathrm{P}}$ and thus $L / \ell_{\mathrm{P}} \sim$ $\sqrt{3 / 8 \pi} \gamma^{-1}$. For this value we have $L / \ell_{\mathrm{P}}>1$, thanks to the smallness of $\gamma \sim 0.24$ as it arises from black hole entropy calculations [44, 45]. This is sufficient to avoid extremely strong inverse volume corrections. However, $L / \ell_{\mathrm{P}} \sim 1.4$ is not much larger than one and inverse volume corrections for this value are still significant. In the specific case of constant $L$ as in [24], the consequences may not be too severe because a constant $L$ implies that inverse volume corrections merely amount to a constant factor in all terms of the constraint where an inverse volume appears. This changes some coefficients, but would leave the qualitative behavior untouched. However, such a parameter choice would be unstable: a slight deviation from a constant patch size $L$, as it can easily occur in general states, would mean that inverse volume corrections become $a$-dependent. Now, it would not only be coefficients but even the qualitative dynamical behavior which is subject to strong changes since, for instance, corrected energy densities $\rho$ containing $\alpha\left(L / \ell_{\mathrm{P}}\right)$ would depend differently on the scale factor than they do classically.

In a strict sense with the usual value of the critical density, we have disproved the improved dynamics of [24]. This dynamics can be consistent with weak inverse volume corrections only if the critical density is much lower than Planckian, and the patch size much larger. For $L$ larger than $\ell_{\mathrm{P}}$ by one order of magnitude, for instance, the critical density could at most be about $2 \%$ of the Planck density.

A constant patch size $v$ or a constant $L$ is only a special case, for which the number of patches is proportional to the volume, $\mathcal{N} \propto a^{3}$. In general, however, both $v$ and $\mathcal{N}$ can be functions of $a$ if the scale factor is used as internal time to measure the change of discrete geometry. As usually, it is often convenient to assume power-law behaviors at least for certain phases of the universe evolution, which we parameterize by a power $x$ in $\mathcal{N}=\mathcal{N}_{0} a^{-6 x}$. The patch size then behaves as

$$
v=a^{3} V_{0} / \mathcal{N}=a^{3(1+2 x)} V_{0} / \mathcal{N}_{0} .
$$

Notice that the parameter $\mathcal{N}_{0}$ in general depends on the size of the region $\mathcal{V}$ as well as coordinates in order to make $\mathcal{N}$ coordinate independent and proportional to $V_{0}$; it is not simply a numerical constant.

In this parameterization, we have $x<0$ if the number of patches $\mathcal{N}$ is increasing, and $x>-1 / 2$ if the patch size $v$ is increasing. Since there is a lower limit for both $\mathcal{N}$ and $v$, values $x>0$ or $x<-1 / 2$ cannot be realized forever. Thus, if a uniform powerlaw model is used, as it is for instance done in solvable models [42, 46, 47, 48] for a free massless scalar, one has to restrict the power to the generic range $-1 / 2<x<0$. The limiting values are possible, but would not be generic as they would require either no change in the patch size to occur $(x=-1 / 2)$ or a constant number of patches $(x=0)$. The former choice, which gives a constant $L$ and thus the dynamics of [24], is impossible in full constructions of loop quantum gravity while the latter is not consistent with largescale semiclassicality. In general, power laws can only be assumed for certain periods of 
time, as it is commonly done for energy densities in different phases of the universe, and occasionally a power $x$ may even fall outside the range $-1 / 2<x<0$. Different powerlaws can then follow each other in much the same way as, e.g., a dust-dominated phase with one power-law for energy density would follow a radiation-dominated phase with a different power-law. Complete functions $\mathcal{N}(a)$ and $v(a)$ incorporating these different phases could only be derived from a full solution of an inhomogeneous state which is difficult, but phenomenological constructions are already possible. See e.g. [49, 50, 51, 52] for earlyuniverse restrictions on $x$ and $L$. As control over the theory increases, one can expect tight consistency conditions to arise in this way.

To restrict general choices even more strongly, anisotropic models are valuable. Effects of lattice refinements, as first analyzed in this context in [33], now allow more freedom because parameters in general depend not only on the volume but also on extensions in different directions. The interior of a Schwarzschild black hole, for instance, is of a Kantowski-Sachs geometry with two independent scales to determine the spatial geometry:

$$
\mathrm{d} s^{2}=\frac{\left(p_{1}\right)^{2}}{\left|p_{2}\right|} \mathrm{d} x^{2}+\left|p_{2}\right| \mathrm{d} \Omega^{2} .
$$

There are two densitized triad components $p_{1}$ and $p_{2}$, which provide the volume $V=$ $4 \pi L_{0}\left|p_{1}\right| \sqrt{\left|p_{2}\right|}$ where $L_{0}$ is a coordinate length parameter to select a finite region whose angular extension has been fixed by using the full sphere size $4 \pi$; for details see [53] or [54]. When triad components and thus the volume vanish, we have either the black hole singularity $\left(p_{2}=0\right)$ or the horizon $\left(p_{1}=0\right)$.

In this context, we can write a more general equation to relate microscopic quantities to the classical geometry:

$$
\mathcal{N}_{1}(t) L(t) \mathcal{N}_{2}(t) A(t)=4 \pi L_{0}\left|p_{1}(t)\right| \sqrt{\left|p_{2}(t)\right|}
$$

where $\mathcal{N}_{1}$ is the number of patches in the $x$-direction, with patch extension $L$ in this direction, and $\mathcal{N}_{2}$ is the number of patches in the spheres spanned by the angular directions, with patch area $A$ in these directions. (Spherical symmetry implies that we do not have to distinguish the two linear extensions in angular directions, which we thus combine in the angular area.)

The volume relation (10) then factorizes in two identities

$$
\mathcal{N}_{1}(t) L(t)=L_{0} \frac{\left|p_{1}(t)\right|}{\sqrt{\left|p_{2}(t)\right|}} \quad, \quad \mathcal{N}_{2}(t) A(t)=4 \pi\left|p_{2}(t)\right|
$$

using the sizes following from the spatial Kantowski-Sachs metric (9) in triad variables. To specify the refinement fully, we have to determine two functions such as $\mathcal{N}_{1}\left(p_{1}, p_{2}\right)$ and $\mathcal{N}_{2}\left(p_{1}, p_{2}\right)$; just the volume dependence of the total number $\mathcal{N}_{1} \mathcal{N}_{2}$ of patches is not sufficient. A particular case, which provides constant patch sizes and shapes, is obtained when $L$ and $A$ are constant and thus $\mathcal{N}_{1} \propto\left|p_{1}\right| / \sqrt{\left|p_{2}\right|}$ and $\mathcal{N}_{2} \propto\left|p_{2}\right|$. This case was introduced in [33] and shown to have stable and near classical behavior in a large region of 
the phase space. It is a special case of models where the total patch number is proportional to volume: $\mathcal{N}_{1} \mathcal{N}_{2} \propto\left|p_{1}\right| \sqrt{\left|p_{2}\right|} \propto V$.

More generally, one can assume a volume dependence $\mathcal{N}_{1} \mathcal{N}_{2} \propto V^{-2 x}$ which still leaves different choices for the anisotropic behavior of the patches. What is important in the present context is that none of these power-law cases can be fully consistent in classical regimes: The total volume vanishes not only at the singularity, where strong discreteness effects would not be unexpected, but also at the horizon where $p_{1}=0$. For a consistent model, which behaves semiclassically near the horizon as it should be the case for massive black holes, one has to use more general functions than power laws to parameterize the refinement behavior. In particular, $\mathcal{N}_{1} \mathcal{N}_{2}$ must not vanish when $p_{1}=0$. Despite many attempts, no fully consistent effective Schwarzschild interior space-time has been constructed yet, even if one restricts oneself to regions far from classical singularities where tree-level equations are reliable and quantum back-reaction effects can be ignored. Anisotropic models thus provide important test cases which are restrictive enough to constrain lattice refinement models further.

To conclude, we have seen that a consistent treatment of loop quantum cosmology, with a robust connection to constructions in the full theory, requires input from the behavior of an underlying microscopic state. If this is done correctly, the balance of quantum corrections changes such that several choices made so far can be ruled out because they would imply strong quantum geometry corrections. (In a pure minisuperspace setting, models with suppressed quantum geometry corrections can be constructed consistently and rather straightforwardly. But they do not consistently model the full theory.) In particular, critical densities must be sufficiently smaller than Planckian, which enlarges holonomy corrections; this may be of interest for potential observations in cosmology because effects, such as those in the tensor mode spectrum [55, 56, 57, 58, 59, would be enlarged. This would have no effect on existing analyses of inverse volume corrections for perturbative inhomogeneities [60, 37, 61] where consistent versions were already used. Since it has become quite fashionable to boldly extend tree-level equations such as (7) and its analogs in black hole models well beyond their proven range of validity and deeply into putative quantum regimes around classical singularities, one should note that this is not done here. To rule out a Planckian critical density we do not have to consider the dynamics near a classical singularity, nor do we have to consider long stretches of evolution which would both give rise to quantum back-reaction in non-solvable models. For a Planckian critical density, consistent inverse volume corrections would be significant at all times even if matter densities are small. In this way, reliable upper bounds on the critical density arise.

\section{Acknowledgements}

This work was supported in part by NSF grant 0748336 . 


\section{References}

[1] C. Rovelli, Quantum Gravity, Cambridge University Press, Cambridge, UK, 2004

[2] A. Ashtekar and J. Lewandowski, Background independent quantum gravity: A status report, Class. Quantum Grav. 21 (2004) R53-R152, gr-qc/0404018

[3] T. Thiemann, Introduction to Modern Canonical Quantum General Relativity, Cambridge University Press, Cambridge, UK, 2007, gr-qc/0110034

[4] M. Bojowald, Loop Quantum Cosmology, Living Rev. Relativity 11 (2008) 4, |gr-qc/0601085], http://www. livingreviews.org//rr-2008-4

[5] N. Weiss, Constraints on Hamiltonian lattice formulations of field theories in an expanding universe, Phys. Rev. D 32 (1985) 3228-3232

[6] W. Unruh, Time, gravity, and quantum mechanics, In: Time's arrows today, Ed.: S. F. Savitt, pages 23-94, gr-qc/9312027

[7] T. Jacobson, Trans-Planckian redshifts and the substance of the space-time river, hep-th/0001085

[8] R. Doldán, R. Gambini, and P. Mora, Quantum mechanics for totally constrained dynamical systems and evolving Hilbert spaces, Int. J. Theor. Phys. 35 (1996) 2057, hep-th/9404169

[9] M. Bojowald, The dark side of a patchwork universe, Gen. Rel. Grav. 40 (2008) 639-660, arXiv:0705.4398

[10] M. Bojowald and A. Skirzewski, Effective Equations of Motion for Quantum Systems, Rev. Math. Phys. 18 (2006) 713-745, math-ph/0511043

[11] M. Bojowald, B. Sandhöfer, A. Skirzewski, and A. Tsobanjan, Effective constraints for quantum systems, Rev. Math. Phys., to appear, arXiv:0804.3365

[12] C. Rovelli and L. Smolin, Loop Space Representation of Quantum General Relativity, Nucl. Phys. B 331 (1990) 80-152

[13] T. Thiemann, Quantum Spin Dynamics (QSD), Class. Quantum Grav. 15 (1998) 839-873, gr-qc/9606089

[14] T. Thiemann, QSD V: Quantum Gravity as the Natural Regulator of Matter Quantum Field Theories, Class. Quantum Grav. 15 (1998) 1281-1314, gr-qc/9705019]

[15] M. Bojowald, Loop quantum cosmology and inhomogeneities, Gen. Rel. Grav. 38 (2006) 1771-1795, gr-qc/0609034 
[16] C. Rovelli and L. Smolin, The physical Hamiltonian in nonperturbative quantum gravity, Phys. Rev. Lett. 72 (1994) 446-449, gr-qc/9308002

[17] K. Giesel and T. Thiemann, Algebraic Quantum Gravity (AQG) I. Conceptual Setup, Class. Quantum Grav. 24 (2007) 2465-2497, gr-qc/0607099

[18] P. Peter and N. Pinto-Neto, Cosmology without inflation, Phys. Rev. D 78 (2008) 063506, arXiv:0809.2022]

[19] M. Bojowald, Isotropic Loop Quantum Cosmology, Class. Quantum Grav. 19 (2002) 2717-2741, gr-qc/0202077

[20] J. F. Barbero G., Real Ashtekar Variables for Lorentzian Signature Space-Times, Phys. Rev. D 51 (1995) 5507-5510, gr-qc/9410014

[21] G. Immirzi, Real and Complex Connections for Canonical Gravity, Class. Quantum Grav. 14 (1997) L177-L181

[22] A. Ashtekar, M. Bojowald, and J. Lewandowski, Mathematical structure of loop quantum cosmology, Adv. Theor. Math. Phys. 7 (2003) 233-268, gr-qc/0304074

[23] C. Rovelli and F. Vidotto, Stepping out of Homogeneity in Loop Quantum Cosmology, Class. Quantum Grav. 25 (2008) 225024, arXiv:0805.4585]

[24] A. Ashtekar, T. Pawlowski, and P. Singh, Quantum Nature of the Big Bang: Improved dynamics, Phys. Rev. D 74 (2006) 084003, |gr-qc/0607039|

[25] P. Dzierzak, J. Jezierski, P. Malkiewicz, and W. Piechocki, Quantum Big Bounce, arXiv:0810.3172

[26] C. Rovelli and L. Smolin, Discreteness of Area and Volume in Quantum Gravity, Nucl. Phys. B 442 (1995) 593-619, gr-qc/9411005], Erratum: Nucl. Phys. B 456 (1995) 753

[27] A. Ashtekar and J. Lewandowski, Quantum Theory of Geometry II: Volume Operators, Adv. Theor. Math. Phys. 1 (1997) 388-429, gr-qc/9711031

[28] M. Bojowald, Inverse Scale Factor in Isotropic Quantum Geometry, Phys. Rev. D 64 (2001) 084018, gr-qc/0105067

[29] J. Brunnemann and T. Thiemann, Simplification of the Spectral Analysis of the Volume Operator in Loop Quantum Gravity, Class. Quantum Grav. 23 (2006) 12891346, gr-qc/0405060

[30] J. Brunnemann and T. Thiemann, Unboundedness of Triad-Like Operators in Loop Quantum Gravity, Class. Quantum Grav. 23 (2006) 1429-1483, gr-qc/0505033

[31] J. Brunnemann and D. Rideout, Properties of the Volume Operator in Loop Quantum Gravity I: Results, Class. Quant. Grav. 25 (2008) 065001, arXiv:0706.0469] 
[32] J. Brunnemann and D. Rideout, Properties of the Volume Operator in Loop Quantum Gravity II: Detailed Presentation, Class. Quant. Grav. 25 (2008) 065002, arXiv:0706.0382

[33] M. Bojowald, D. Cartin, and G. Khanna, Lattice refining loop quantum cosmology, anisotropic models and stability, Phys. Rev. D 76 (2007) 064018, arXiv:0704.1137]

[34] M. Bojowald, Quantization ambiguities in isotropic quantum geometry, Class. Quantum Grav. 19 (2002) 5113-5130, gr-qc/0206053]

[35] M. Bojowald, Loop Quantum Cosmology: Recent Progress, Pramana 63 (2004) 765776, In Proceedings of the International Conference on Gravitation and Cosmology (ICGC 2004), Cochin, India, gr-qc/0402053

[36] M. Bojowald, H. Hernández, M. Kagan, and A. Skirzewski, Effective constraints of loop quantum gravity, Phys. Rev. D 75 (2007) 064022, gr-qc/0611112

[37] M. Bojowald, G. Hossain, M. Kagan, and S. Shankaranarayanan, Anomaly freedom in perturbative loop quantum gravity, Phys. Rev. D 78 (2008) 063547, arXiv:0806.3929

[38] M. Bojowald, H. Hernández, and A. Skirzewski, Effective equations for isotropic quantum cosmology including matter, Phys. Rev. D 76 (2007) 063511, arXiv:0706.1057]

[39] M. Bojowald, How quantum is the big bang?, Phys. Rev. Lett. 100 (2008) 221301, arXiv:0805.1192

[40] P. Singh, Loop cosmological dynamics and dualities with Randall-Sundrum braneworlds, Phys. Rev. D 73 (2006) 063508, [gr-qc/0603043]

[41] M. Bojowald, Quantum nature of cosmological bounces, Gen. Rel. Grav. (2008) to appear, arXiv:0801.4001

[42] M. Bojowald, Large scale effective theory for cosmological bounces, Phys. Rev. D 75 (2007) 081301(R), gr-qc/0608100

[43] O. Hrycyna, J. Mielczarek, and M. Szydłowski, Effects of the quantisation ambiguities on the Big Bounce dynamics, arXiv:0804.2778]

[44] M. Domagala and J. Lewandowski, Black hole entropy from Quantum Geometry, Class. Quantum Grav. 21 (2004) 5233-5243, gr-qc/0407051]

[45] K. A. Meissner, Black hole entropy in Loop Quantum Gravity, Class. Quantum Grav. 21 (2004) 5245-5251, [gr-qc/0407052]

[46] M. Bojowald, Dynamical coherent states and physical solutions of quantum cosmological bounces, Phys. Rev. D 75 (2007) 123512, gr-qc/0703144]

[47] M. Bojowald, What happened before the big bang?, Nature Physics 3 (2007) 523-525 
[48] M. Bojowald, Harmonic cosmology: How much can we know about a universe before the big bang?, Proc. Roy. Soc. A 464 (2008) 2135-2150, arXiv:0710.4919

[49] W. Nelson and M. Sakellariadou, Lattice Refining Loop Quantum Cosmology and Inflation, Phys. Rev. D 76 (2007) 044015, arXiv:0706.0179.

[50] W. Nelson and M. Sakellariadou, Lattice Refining LQC and the Matter Hamiltonian, Phys. Rev. D 76 (2007) 104003, arXiv:0707.0588

[51] M. Bojowald and G. Hossain, Quantum gravity corrections to gravitational wave dispersion, Phys. Rev. D 77 (2008) 023508, arXiv:0709.2365.

[52] M. Bojowald, R. Das, and R. Scherrer, Dirac fields in Loop Quantum Gravity and Big Bang Nucleosynthesis, Phys. Rev. D 77 (2008) 084003, arXiv:0710.5734]

[53] A. Ashtekar and M. Bojowald, Quantum Geometry and the Schwarzschild Singularity, Class. Quantum Grav. 23 (2006) 391-411, gr-qc/0509075

[54] L. Modesto, Loop quantum black hole, Class. Quantum Grav. 23 (2006) 5587-5601, gr-qc/0509078

[55] A. Barrau and J. Grain, Holonomy corrections to the cosmological primordial tensor power spectrum, arXiv:0805.0356

[56] E. J. Copeland, D. J. Mulryne, N. J. Nunes, and M. Shaeri, The gravitational wave background from super-inflation in Loop Quantum Cosmology, arXiv:0810.0104.

[57] J. Mielczarek, Gravitational waves from the Big Bounce, arXiv:0807.0712]

[58] G. Calcagni and G. Hossain, Loop quantum cosmology and tensor perturbations in the early universe, Adv. Sci. Lett. (2008) to appear, arXiv:0810.4330

[59] A. Barrau and J. Grain, Cosmological footprint of loop quantum gravity, in preparation

[60] M. Bojowald, H. Hernández, M. Kagan, P. Singh, and A. Skirzewski, Formation and evolution of structure in loop cosmology, Phys. Rev. Lett. 98 (2007) 031301, astro-ph/0611685

[61] M. Bojowald, G. Hossain, M. Kagan, and S. Shankaranarayanan, Gauge invariant cosmological perturbation equations with corrections from loop quantum gravity, arXiv:0811.1572 